\title{
Analysis of Two Dimensional Circuits Based on Multi-Conductor Theorem
}

\author{
Akio USHIDA, Yuichi TANJI and Yoshifumi NISHIO \\ Department of Electrical and Electronic Engineering, \\ Tokushima University, Tokushima, 770 JAPAN \\ Tel.: +81-886-56-7469, Fax.: +81-886-56-7471 \\ E-mail: ushida@ee.tokushima-u.ac.jp
}

\section{ABSTRACT}

We discuss an efficient algorithm for solving 2-dimensional circuits based on a multi-conductor theorem. There have been many papers published about transmission lines, because they are very important for design of high speed VLSI chips. On the other hand, a device simulation is also very important to design ICs and to understand the qualitative behavior. Most of the simulation techniques are based on the finite-difference time-domain method, where the devices are described by many discrete models. This is really time-consuming because, to get exact solution, the device must be divided into many sections. In this paper, we show an elegant algorithm for solving 2-dimensional circuits, which can be applied to the device simulations.

\section{INTRODUCTION}

Most of device simulations are based on the finite element techniques. Recently, new methods have been proposed for analyizing 2-dimensional circuits, where the linearization of the discretized semiconductor equations [1-2] permits the efficient solution for either transient or steady-state. For three dimensional model, the devices are divided into much more pieces so that we need to solve large scale equations and matrices. The reference [3] has proposed an efficient technique using parallel computation technique. As one of the applications, it can be used to estimate the resistance between two points on the plate, where two efficient techniques are proposed. One of them is based on a boundary element method [4], and the other is a node elimination technique [5].

For conventional semiconductor devices, the circuit equations can be described by partial differential equations, and are usually solved by the finite element techniques. It is really time-consuming, so that many efficient techniques are proposed [6-8]. Mixed-level circuit and device simulations [7-8] are also time-consuming task, because we must solve both device and circuit at every time point.

However, for a small input signal, the device is linearized around the dc operation point so that we can solve it in the frequency-domain [7].

In this paper, we propose an elegant method for solving 2-dimensional distributed circuits based on the multiconductor theorem. At first, we discretize the plane circuit into two directions such that each transmission line is connected by the discretized R-L elements in each other. We found that after some transformations, the circuit equation is also described by the form of multi-conductor transmission lines. Thus, it can be efficiently solved in the time-domain [9-10] and the frequency-domain [11-12] techniques.

Note that when 2-dimensional circuit is discretized in $M \times N$ pieces, we must solve the same number of the circuit equations. The discretizing technique is really timeconsuming for larger $M$ and $N$. Our multi-conductor method only takes into account $N$ or $M$ lines, so that the computational efficiency is largely improved in compared with the usual discretized methods. As an illustrated example, we solved a 2-dimensional circuit composed of 2 different plates.

\section{Analysis of 2-Dimensional Circuits}

In order to analyze the two-dimensional circuits, we discretize the circuit into the $x$ and $y$ axes as shown in Fig.1.

Thus, the circuit equation can be described by the following partial equations:

$$
\begin{aligned}
& \frac{\partial v_{i}}{\partial x}=-\left(L_{m} \frac{\partial i_{i-1}}{\partial t}+L_{x} \frac{\partial i_{i}}{\partial t}+L_{m} \frac{\partial i_{i+1}}{\partial t}\right) \\
&-\left(R_{m} i_{i-1}+R_{x} i_{i}+R_{m} i_{i+1}\right) \\
& \frac{\partial i_{i}}{\partial x}=-\left(C_{m} \frac{\partial v_{i-1}}{\partial t}+C_{x} \frac{\partial v_{i}}{\partial t}+C_{m} \frac{\partial v_{i+1}}{\partial t}\right) \\
&-\left(G_{m} v_{i-1}+G_{x} v_{i}+G_{m} v_{i+1}\right) \\
&+i_{y, i}-i_{y, i-1}
\end{aligned}
$$




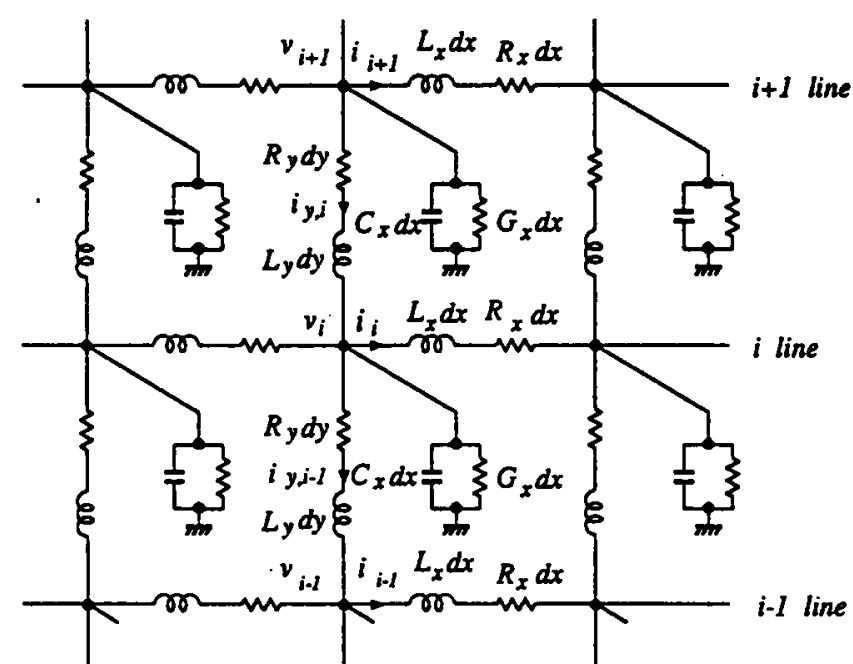

Fig.1 Discretized model of 2-dimensional circuit

$$
\begin{gathered}
v_{i}-v_{i-1}=L_{y} \frac{\partial i_{y, i-1}}{\partial t}+R_{y} i_{y, i-1} \\
v_{i+1}-v_{i}=L_{y} \frac{\partial i_{y, i}}{\partial t}+R_{y} i_{y, i} \\
i=1,2, \ldots, n
\end{gathered}
$$

where $n$ denotes the total lines, and $\left(L_{x}, R_{x}, C_{x}, G_{x}\right)$ and $\left(L_{m}, R_{m}, C_{m}, G_{m}\right)$ are self-impedances and couplingimpedances between the lines, respectively. The circuit equation is coupled by (1.3) and (1.4), and different from those of multi-conductor transmission lines [11]. Although there are time-domain and frequency-domain approaches for solving transmission lines, we apply the frequencydomain technique [12], because it can be easily applied to both $\mathrm{AC}$ and transient analysis of 2-dimensional circuits. Now, consider the responses of the following impulse waveform:

$$
\begin{aligned}
e(t) & =E_{m} \sin (K t) & & : 0 \leq t \leq T / K \\
& =0 & & : T / K \leq t \leq T
\end{aligned}
$$

and we expand it in the Fourier series as follows:

$$
e(t)=E_{0}+\sum_{k=1}^{M}\left(E_{2 k-1} \cos k \omega t+E_{2 k} \sin k \omega t\right)
$$

where $\omega=2 \pi / T$. Assume

$$
v_{i}=V_{i} e^{j k \omega t}, i_{i}=I_{i} e^{j k \omega t}, i_{y i}=I_{y i} e^{j k \omega t}
$$

and substituting these relations into (1), we have

$$
\frac{d V}{d x}=-(R+j k \omega L) I, \quad \frac{d I}{d x}=-(G+j k \omega B) V
$$

where each of $R, X, G$ and $B$ matrices has the same form as follow: i.e. let $A$ be an arbitrary matrix of $R, X, G$ and $B$. Then, we have

$$
A=\left(\begin{array}{ccccc}
a_{x} & a_{m} & & & 0 \\
a_{m} & a_{x} & a_{m} & & \\
& \cdots & \cdots & & \\
& & \cdots & \cdots & \\
0 & & a_{m} & a_{x} & a_{m} \\
0 & & & a_{m} & a_{x}
\end{array}\right)
$$

where the tridiagonal elements of $G$ and $B$ in (4) given by

$$
\begin{gathered}
G_{x}+j k \omega B_{x} \equiv\left(G_{x}+\frac{2 R_{y}}{R_{y}^{2}+\left(k \omega L_{y}\right)^{2}}\right) \\
+j k \omega\left(C_{x}-\frac{2 L_{y}}{R_{y}^{2}+\left(k \omega L_{y}\right)^{2}}\right) \\
G_{m}+j k \omega B_{m} \equiv\left(G_{m}-\frac{R_{y}}{R_{y}^{2}+\left(k \omega L_{y}\right)^{2}}\right) \\
+j k \omega\left(C_{m}+\frac{L_{y}}{R_{y}^{2}+\left(k \omega L_{y}\right)^{2}}\right)
\end{gathered}
$$

Thus, $A$ is a tridiagonal and symmetric Toeplitz matrix, so that the linear transformation is easily achieved. It is known that the transfomation matrix $M$ is independent of the element $A$, and the inverse of $M$ is equal to the transpose of it [11]. Furthermore, the eigenvalues are given as

$$
\lambda_{i}=a_{z}+a_{m} \mu
$$

The elements of matrix $M$ can be computed as

$$
m_{i, j}=\phi_{i-1}\left(\mu_{i}\right) / \delta_{j}
$$

where $\phi_{i}(\mu)$ is given in the form

$$
\dot{\phi}_{i}(\mu)=\mu \phi_{i-1}(\mu)-\phi_{i-2}(\mu), \quad i \geq 2
$$

The initial conditions are defined as: $\phi_{0}(\mu)=1$ and $\phi_{1}(\mu)=\mu$, and the normalization factor $\delta_{i}$ is given by

$$
\delta_{i}^{2}=\sum_{i=1}^{n}\left(\phi_{i-1}\left(\mu_{i}\right)\right)
$$

It can be shown that the transformation matrix of the tridiagonal symmetric matrices has its inverse matrix equal to its transpose matrix.

Using $M$, let us transform relations (4) as follows:

$$
V=M V^{\prime}, \quad I=M I^{\prime}
$$

Thus, we have

$$
\frac{d V^{\prime}}{d x}=-M^{T} R M I^{\prime}-j k \omega M^{T} X M I^{\prime}
$$




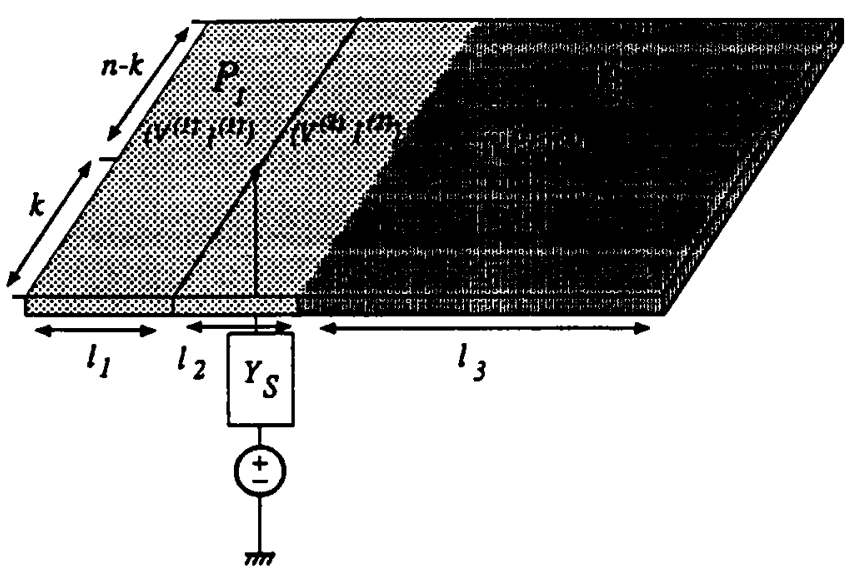

Fig.2 2-dimensional circuit

$$
\frac{d I^{\prime}}{d x}=-M^{T} G M V^{\prime}-j k \omega M^{T} B M V^{\prime}
$$

All of the right hand side can be described by the diagonal matrices as follows:

$$
\begin{aligned}
& \frac{d V_{i}^{\prime}}{d x}=-\left(r_{i, i}+j k \omega x_{i, i}\right) I_{i}^{\prime} \\
& \frac{d I_{i}^{\prime}}{d x}=-\left(g_{i, i}+j k \omega b_{i, i}\right) V_{i}^{\prime}
\end{aligned}
$$

Thus, the solution is given by

$$
\begin{gathered}
V_{i}^{\prime}=K_{1, i} e^{\gamma_{i, i} x}+K_{2, i} e^{-\gamma_{i, i} x} \\
I_{i}^{\prime}=\frac{1}{Z_{i, i}}\left(-K_{1, i} e^{\gamma_{i, i} x}+K_{2, i} e^{-\gamma_{i, i} x}\right)
\end{gathered}
$$

where the characteristic impedance and propagation constant are given by

$$
\begin{gathered}
Z_{i, i}=\sqrt{\frac{r_{i, i}+j k \omega x_{i, i}}{g_{i, i}+j k \omega b_{i, i}}} \\
\gamma_{i, i}=\sqrt{\left(r_{i, i}+j k \omega x_{i, i}\right)\left(g_{i, i}+j k \omega b_{i, i}\right)}
\end{gathered}
$$

where the constants $K_{1, i}$ and $K_{2, i}$ are decided by the boundary value conditions of the 2 -dimensional plate as shown in Fig.2, which is composed of two different plates $P_{1}\left(\gamma_{i, i}^{(1)}, Z_{i, i}^{(1)}\right)$, and $P_{2}\left(\gamma_{i, i}^{(2)}, Z_{i, i}^{(2)}\right)$. Assume the input location is $k$-th line and $l_{1}$ from the left end, and choose the origin at $\left(l_{1}, k\right)$. Then, we have the following 6 boundary conditions:

1. Current at the left end is zero: $I^{(1)}\left(l_{1}\right)=0$

$$
\Rightarrow-\operatorname{diag}\left(\frac{e^{\gamma_{i, i}^{(1)} l_{1}}}{Z_{i, i}^{(1)}}\right) K_{1}^{(1)}+\operatorname{diag}\left(\frac{e^{-\gamma_{i, i}^{(1)} l_{1}}}{Z_{i, i}^{(1)}}\right) K_{2}^{(1)}=0
$$

2. The current must be continuous at the bound. ary of $P_{1}$ and $P_{2}: I^{(2)}\left(l_{2}\right)=I^{(3)}(0)$

$$
\begin{gathered}
\Rightarrow-\operatorname{diag}\left(\frac{e^{\gamma_{i, i}^{(1)} l_{2}}}{Z_{i, i}^{(1)}}\right) K_{1}^{(2)}+\operatorname{diag}\left(\frac{e^{-\gamma_{i, i}^{(1)} l_{2}}}{Z_{i, i}^{(1)}}\right) K_{2}^{(2)} \\
=-\operatorname{diag}\left(\frac{1}{Z_{i, i}^{(2)}} K_{1}^{(3)}+\frac{1}{Z_{i, i}^{(2)}} K_{2}^{(3)}\right)
\end{gathered}
$$

3. The voltage must be continuous at the boundary of $P_{1}$ and $P_{2}: V^{(2)}\left(l_{2}\right)=V^{(3)}(0)$

$\Rightarrow \operatorname{diag}\left(e^{\gamma_{i, i}^{(1)} l_{2}}\right) K_{1}^{(2)}+\operatorname{diag}\left(e^{-\gamma_{i, i}^{(1)} l_{2}}\right) K_{2}^{(2)}=K_{1}^{(3)}+K_{2}^{(3)}$

4. Current at the right end is zero: $I^{(3)}\left(l_{3}\right)=0$

$$
\Rightarrow-\operatorname{diag}\left(\frac{e^{\gamma_{i, i}^{(2)} l_{3}}}{Z_{i, i}^{(2)}}\right) K_{1}^{(3)}+\operatorname{diag}\left(\frac{e^{-\gamma_{i, i}^{(2)} l_{2}}}{Z_{i, i}^{(2)}}\right) K_{2}^{(3)}=0
$$

5. Voltage at input line: $V^{(1)}(0)=V^{(2)}(0)$

$$
\Rightarrow K_{1}^{(1)}+K_{2}^{(1)}=K_{1}^{(2)}+K_{2}^{(2)}
$$

6. Current at input line:

$$
\left(\begin{array}{lllll}
Z_{L} & \mid & Z_{L} & \mid & D
\end{array}\right)\left(\begin{array}{c}
I^{(1)}(0) \\
I^{(2)}(0) \\
V^{(1)}(0)
\end{array}\right)=E_{k}
$$

where $Z_{L}$

$$
\begin{aligned}
& =\left(\begin{array}{ccccc}
Z_{y} & 0 & 0 & \cdots & 0 \\
Z_{y} & Z_{y} & 0 & \cdots & 0 \\
\ldots \ldots & \ldots & \ldots & \cdots & \ldots \\
Z_{y} & Z_{y} & Z_{y} & \cdots & 0 \\
Z_{y} & Z_{y} & Z_{y} & \cdots & Z_{y}
\end{array}\right), E_{k}=\left(\begin{array}{c}
0 \\
\cdot \\
Z_{y} Y_{s} E_{k, \text { in }} \\
\cdot \\
Z_{y} Y_{s} E_{k, i n}
\end{array}\right){ }^{1} \\
& D=\left(\begin{array}{ccccc}
1 & -1 & 0 & \cdots & 0 \\
0 & 1 & -1 & \cdots & 0 \\
\cdots & \ldots & \cdots & \cdots & \cdots \\
0 & 0 & 0 & \cdots & -1 \\
0 & 0 & 0 & \cdots & 1
\end{array}\right) \\
& +\left(\begin{array}{ccccc}
0 & \cdots & 0 & \cdots & 0 \\
0 & \cdots & 0 & \cdots & 0 \\
0 & \cdots & \Delta & \cdots & 0 \\
\cdots \cdots \cdots & \cdots & 1 \\
0 & \cdots & \Delta & \cdots & 0
\end{array}\right)_{n} \\
& 1 \ldots k \ldots k
\end{aligned}
$$

Note that the coupling impedance is given by

$$
Z_{y}=R_{y}+j k \omega L_{y}, \quad \Delta=Z_{y} Y_{S}
$$




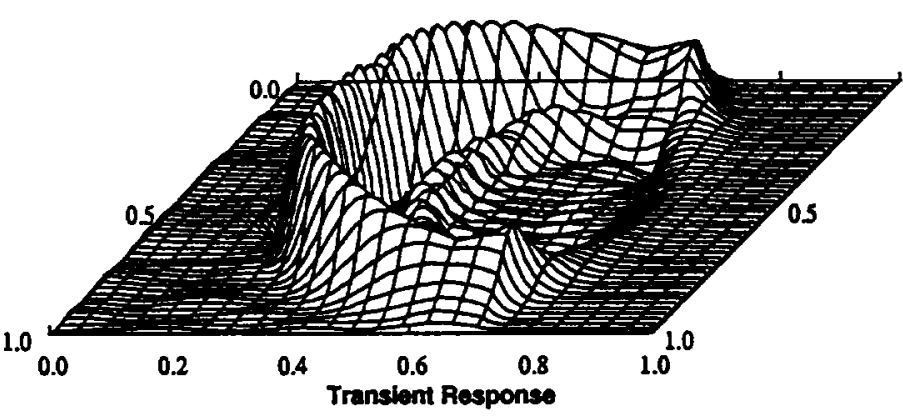

Fig. 2-dimensional circuit

Plate : $48[\mathrm{~mm}] \times 50[\mathrm{~mm}]$

$R_{1}=30[\Omega / \mathrm{mm}] \quad R_{m 1}=1[\Omega / \mathrm{mm}] \quad L_{1}=0.003[\mu / \mathrm{mm}]$

$L_{m l}=0.0001[\mu / m m] \quad G_{1}=30[S / m m] \quad G_{m 1}=-1[S / m m]$

$C_{1}=0.050[\mathrm{pF} / \mathrm{mm}] \quad C_{\mathrm{ml}}=-0.001[\mathrm{pF} / \mathrm{mm}] \quad R_{2}=200[\Omega / \mathrm{mm}]$

$R_{m 2}=10[\Omega / \mathrm{mm}] \quad L_{2}=0.003[\mu / \mathrm{mm}] \quad L_{m 2}=0.0001[\mu / \mathrm{mm}]$

$G_{2}=200[S / \mathrm{mm}] \quad G_{m 2}=-1[S / \mathrm{mm}] \quad C_{2}=0.050[\mathrm{pF} / \mathrm{mm}]$

$C_{m 2}=-0.001[\mathrm{pF} / \mathrm{mm}], \quad R_{s 1}=10[\Omega / \mathrm{mm}] \quad L_{s 1}=0.003[\mu / \mathrm{mm}]$

$R_{\mathrm{s} 2}=200[\Omega / \mathrm{mm}], \quad L_{\mathrm{s} 2}=0.003[\mu / \mathrm{mm}]$

Input position $(30[\mathrm{~mm}], 25[\mathrm{~mm}])$, Time $=0.25[\mathrm{nsec}]$

and $Y_{s}, E_{k, \text { in }}$ are input admittance and the Fourier coefficients of the input impulse.

Solving relations $(10)$, we can estimate the coefficients $\left(K_{1}^{(1)}, K_{2}^{(1)}\right),\left(K_{1}^{(2)}, K_{2}^{(2)}\right)$ and $\left(K_{1}^{(3)}, K_{2}^{(3)}\right)$. Thus, the steady-state waveform at any point can be calculated by the phasor technique.

As an illustrative example, we calculated the impulse response on the two different plates as shown in Fig. 2. We consider here 50 lines in $Y$-directions located $1[\mathrm{~mm}]$ each other, and connected by $R L$ circuit, where $l_{1}=30[\mathrm{~mm}]$, $l_{2}=4[\mathrm{~mm}]$ and $l_{3}=14[\mathrm{~mm}]$. The response waveform at $0.25[\mathrm{nsec}]$ has two reflections from the boundary of two plates and the right end as shown in Fig.3.

\section{Conclusions ANd REMARKS}

We proposed here an elegant algorithm for solving 2dimensional circuits based on a multi-conductor transmission line theorem. Since the transfer matrix obtained by the use of Toeplitz's theorem is very simple, it can be easily applied to the large scale circuits. As an illustrative example, we solved 2-dimensional circuit composed of two different plates. The computational efficiency is largely improved compared to the usual finite element technique. Furthermore, the method can be also applied to solve 3dimensional circuits and devices. Thus, the technique will be used for wide classes of device simulations.

\section{REFERENCES}

[1] M.S. Obrecht,"A new stable method for linearization of discretized basic semiconductor equation, "Solid State Electron., vol.36, no.4, pp.643-648,1993.

[2] M.S. Obrecht, M.I. Elmasry and E.L. Heasell, "TRASIM: Compact and efficient two-dimensional transient simulator for arbitrary planner semiconductor devices," IEEE Trans. on Computer-Aided Design, vol.14, no.4, pp.447458, 1995.

[3] S. Odanaka and T. Nogi, "Massively parallel Computation using a splitting-up operator method for three-dimensional device simulation," IEEE Trans. on Computer-Aided Design, vol.14, no.7, pp.824-832, 1995.

[4] Z. Wang and $Q$. Wu, "A two-dimensional resistance simulator using the boundary element method," IEEE Trans. on Computer-Aided Design, vol.11, no.4, pp.497$504,1992$.

[5] T. Mitsuhashi and K. Yoshida, "A resistance calculation algorithm and its application to circuit extraction," IEEE Trans. on Computer-Aided Desigm, vol.6, no.3, pp.337$345,1987$.

[6] S.P. Chin and C.Y. Wu, "A new grid-generation method for 2-D simulation of devices with nonplaner semiconductor surface," IEEE Trans. on Computer-Aided Design, vol.12, no.9, pp.1337-1344, 1993.

17] K. Mayaram and D.O. Pederson, "Coupling algorithm for mixed-level circuit and device simulation," IEEE Trans. on Computer-Aided Design, vol.11, no.8, pp.1003-1012, 1992.

[8] N. Iwamuro and S. Tagami, "Two-dimensional power device simulator considering an integral external circuit equation," IEEE Trans. on Computer-Aided Design, vol.12, no.6, pp.909-912, 1993.

[9] L.Lu, N. Nakhla and Q.J. Zhang, "A resetting algorithm for transient analysis of coupled transmission lines," IEEE Trans. on Microwave Theory and Tech., vol.42, no.3, pp.494-500, 1994.

[10] F.Y. Chang, "Transient simulation of nonuniform coupled lossy transmission lines characterized with frequencydependent parameters Part II:Discrete -time analysis,", IEEE Trans. on Circuits and Syst: Fundamental Theory and Applications, vol.39, no.11, pp.907-927, 1992.

[11] D.S. Gao, A.T. Yang and S.M. Kang, "Modeling and simulation of interconnection delays and crosstalks in highspeed integrated circuits," IEEE Trans. on Circuits and Syst., vol.37, no.1, pp.1-9, 1990.

[12] Y. Tanji, L. Jiang and A. Ushida, "Analysis of pulse responses of multi-conductor transmission lines by a partitioning technique," IEICE Trans. on Fundamentals, vol.E77-A, no.12, pp.2017-2027, 1994. 